\title{
GEOMETRY OF GROUP REPRESENTATIONS
}

\author{
G. DE B. ROBINSON
}

To the memory of TADASI NAKAYAMA

The many unanswerable questions (1) which arise in the study of finite groups have lead to a review of fundamental ideas, e.g. the Theorem of Burnside $(3$, p. $299 ; 2,6)$ that if $\lambda$ be any faithful irreducible representation of $G$ over a field $K$, then every irreducible representation of $G$ over $K$ is contained in some tensor power of $\lambda$.

If we take $K$ to be the complex field and write the inner tensor product in question $\lambda \times \lambda \times \cdots$ ( $n$ factors) as $\lambda \times{ }^{n}$, we recall Schur's result that this representation of $G$ splits according to the formula (7, p. 129)

$$
\lambda \times{ }^{n}=\sum f_{\nu} \lambda \otimes[\nu]
$$

where $\lambda \otimes[\nu]$ is the symmetrized inner product associated with the irreducible representation $[\nu]$ of degree $f_{v}$ of the symmetric group $S_{n}$. For a finite group $G, \lambda \otimes[\nu]$ is in general reducible, while for the full linear group and certain of its subgroups this representation is irreducible.

These symmetrized tensor products are hard to handle, though their degrees $\delta^{\nu}$ are given by the formula $(5$, p. 60$)$

$$
1.2 \quad \delta^{\nu}\left(f_{\lambda}\right)=G^{\nu}\left(f_{\lambda}\right) / H^{\nu}
$$

where $f_{\lambda}$ is the degree of $\lambda$. If we denote the Young diagram associated with the irreducible representation $\nu$ of $S_{n}$ by $[\nu]$, then $H^{\nu}$ is the product of hook length of $[\nu]$ and $G^{\prime}\left(f_{\lambda}\right)=\prod_{i, j}\left(f_{\lambda}+j-i\right)$, taken over [ $\left.\nu\right]$. It follows immediately that for $n \leq f_{\lambda}$, all these symmearized products are defined.

It would be interesting if Burnside's theorem could be refined so as to relate the apperances of the different irreducible representations of $G$ to these symmetrized components of $\lambda \times^{n}$, but the difficulties seem insurmountable at present.

Received May 14, 1965. 
2. Another application of these tensor products is of interest. In Chapter XII of (3) Burnside studies at some length the permutation representation $g_{i}$ of $G$ induced by the identity representation of a subgroup $H_{i}(i=1,2, \ldots, r)$ of orders $h_{i}$. It is natural to arrange the $H_{i}$ so that $H_{1}=I$ and $g_{1}$ is the regular representation of $G, h_{i} \leq h_{i+1} \leq h_{r}$ with $H_{r}=G$ so that $g_{r}$ is the identity representation of $G$. If we suppose $g_{i}$ to be represented on the variables $x_{u}$ and $g_{j}$ on the variables $y_{v}$, the tensor product $g_{i} \times g_{j}$ is represented on the variables $x_{u} y_{v}$ and

$$
g_{i} \times g_{j}=\sum a_{i j k} g_{k} .
$$

If $j=i$, we obtain the symmetrized components for $n=2$ on the variables (5, p. 57 ).

$$
x_{1} y_{1}, x_{2} y_{2}, \ldots, \frac{1}{2}\left(x_{u} y_{v}+x_{v} y_{u}\right) ; \ldots \frac{1}{2}\left(x_{u} y_{v}-x_{v} y_{u}\right)
$$

by setting $y_{u}=x_{\boldsymbol{u}}$. It follows, as in the case of $g_{i} \times g_{j}$, that $g_{i} \otimes[2]$ is also $a$ permutation representation of $G$, while $g_{i} \otimes\left[1^{2}\right]$ is not. The argument is quite general so that 2.1 becomes

$$
g_{i} \times^{n}=\sum_{j} a_{i j}^{n} g_{j}
$$

and we have

$$
g_{i} \otimes[n]=\sum_{j} b_{i j}^{n} g_{j},
$$

where the $a_{i j}^{n}, b_{i j}^{n}$ are rational integers.

3. What is of interest here is that $2.1-2.3$ can be interpreted in a natural way relative to the geometry of the irreducible representations $\lambda$ of $G$. A start was made on this many years ago (4). For purposes of illustration, we reproduce two tables which set the stage for this interpretation in the case of $S_{4}$. Here we write

$$
g_{i}=\sum_{\nu} m_{i}^{\nu}[\nu]
$$

and Table 2 gives the values of the $m_{i}^{\nu}$. For completeness, it would have been desirable to list all the solutions of 2.1 , but this has been omitted in favour of Table 3 which gives the solutions of 2.2 and 2.3 for $n=2,3$. Since there are five irreducible representations of $S_{1}$, we have the following linear relations between the $g_{i}$ : 
TABLE 1

\begin{tabular}{llr}
\hline$H$ & \multicolumn{1}{c}{ sub-group } & $h$ \\
\hline$H_{1}$ & $1,(12)$ & 1 \\
$H_{2}$ & $1,(12)(34)$ & 2 \\
$H_{3}$ & $1,(123),(132)$ & 2 \\
$H_{4}$ & $1,(123),(13)(24),(1432)$ & 3 \\
$H_{5}$ & $1,(1234)(34),(14)(23),(13)(24)$ & 4 \\
$H_{6}$ & $1,(12)(34),(34),(12)(34)$ & 4 \\
$H_{7}$ & $1,(12),(12),(132)$ & 4 \\
$H_{8}$ & $1,(12),(13),(23),(123),(132),(13)(24),(1324),(1423)$ & 8 \\
$H_{9}$ & $1,(12),(34),(12)(34),(14)(23),(12$ \\
$H_{10}$ & $A_{4}$ & 24 \\
$H_{11}$ & $S_{4}$ & 12 \\
\hline
\end{tabular}

TABLE 2

\begin{tabular}{|c|c|c|c|c|c|}
\hline & {$\left[1^{4}\right]$} & {$\left[2,1^{2}\right]$} & {$\left[2^{2}\right]$} & {$[3.1]$} & [4] \\
\hline$g_{1}$ & 1 & 3 & 2 & 3 & 1 \\
\hline$g_{2}$ & . & 1 & 1 & 2 & 1 \\
\hline$g_{3}$ & 1 & 1 & 2 & 1 & 1 \\
\hline$g_{4}$ & 1 & 1 & . & 1 & $\hat{1}$ \\
\hline$g_{5}$ & • & 1 & 1 & $\bullet$ & 1 \\
\hline$g_{6}$ & 1 & • & 2 & • & 1 \\
\hline$g_{7}$ & • & • & 1 & 1 & 1 \\
\hline$g_{8}$ & • & • & • & 1 & 1 \\
\hline $\begin{array}{l}g_{9}^{\circ}\end{array}$ & • & • & 1 & • & 1 \\
\hline$g_{10}$ & 1 & • & $\bullet$ & • & 1 \\
\hline \multirow[t]{2}{*}{$g_{11}$} & - & • & $\bullet$ & $\bullet$ & 1 \\
\hline & & & $m_{i}^{\nu}$ & & \\
\hline
\end{tabular}

TABLE 3

\begin{tabular}{lllll}
\hline & \multicolumn{1}{c}{$\times^{2}$} & \multicolumn{1}{c}{$\times^{3}$} & \multicolumn{1}{c}{$\otimes[2]$} & $\otimes[3]$ \\
\hline$g_{1}$ & $24 g_{1}$ & $576 g_{1}$ & $8 g_{1}+6 g_{2}+3 g_{3}$ & $17 g_{1}+4 g_{4}$ \\
\hline$g_{2}$ & $5 g_{1}+2 g_{2}$ & $70 g_{1}+4 g_{2}$ & $g_{1}+4 g_{2}+g_{6}$ & $11 g_{1}+7 g_{2}+2 g_{4}$ \\
\hline$g_{3}$ & $4 g_{1}+4 g_{3}$ & $64 g_{1}+16 g_{3}$ & $3 g_{2}+3 g_{3}+g_{5}$ & $10 g_{1}+9 g_{3}+2 g_{4}$ \\
\hline$g_{4}$ & $2 g_{1}+2 g_{4}$ & $20 g_{1}+4 g_{4}$ & $g_{2}+g_{3}+g_{4}+g_{8}$ & $4 g_{1}+3 g_{4}$ \\
\hline$g_{5}$ & $g_{1}+2 g_{5}$ & $8 g_{1}+4 g_{5}$ & $g_{2}+g_{5}+g_{9}$ & $g_{1}+g_{3}+g_{4}+g_{5}$ \\
\hline$g_{6}$ & $6 g_{6}$ & $36 g_{6}$ & $3 g_{6}+g_{9}$ & $9 g_{6}+g_{10}$ \\
\hline$g_{7}$ & $g_{1}+2 g_{7}$ & $8 g_{1}+4 g_{7}$ & $g_{2}+g_{7}+g_{9}$ & $g_{1}+g_{3}+2 g_{7}+2 g_{8}$ \\
\hline$g_{8}$ & $g_{2}+g_{8}$ & $g_{1}+3 g_{2}+g_{8}$ & $g_{7}+g_{8}$ & $g_{2}+2 g_{8}$ \\
\hline$g_{9}$ & $g_{6}+g_{9}$ & $4 g_{6}+g_{9}$ & $2 g_{9}$ & $g_{6}+g_{9}+g_{11}$ \\
\hline$g_{10}$ & $2 g_{10}$ & $4 g_{10}$ & $g_{10}+g_{11}$ & \\
\hline$g_{11}$ & $g_{11}$ & $g_{11}$ & & \\
\hline
\end{tabular}




$$
\begin{array}{ll}
2 g_{6}+g_{1}=3 g_{3} & 2 g_{9}+g_{1}=g_{2}+g_{3}+g_{5} \\
2 g_{7}+g_{1}=2 g_{2}+g_{3} & 2 g_{10}+g_{1}=g_{3}+2 g_{4} \\
2 g_{8}+g_{1}=2 g_{2}+g_{4} & 2 g_{11}+g_{1}=g_{2}+g_{4}+g_{5}
\end{array}
$$

Consider, in particular the irreducible representation $[3,1]$ whose invariant configuration is a regular tetrahedron. Since $H_{4} \subset H_{8}$, the groups of stability of the vertices are $H_{3}$ and its conjugates. Taking the bi-vector defined by two such vertices, we have from Table 3 ,

$$
g_{8} \times{ }^{2}=g_{8}+g_{2}
$$

which indicates that the group of stability of the corresponding edge is $H_{2}$ with $m_{2}^{[3,1]}=2$. However, this does not take into account the extra symmetry arising by interchanging the two vertices. For this we go to

$$
g_{*} \otimes[2]=g_{8}+g_{i},
$$

and the group of stability of the mid-edge point is $H_{7}$. As already mentioned, the component

$$
g_{8} \otimes\left[1^{2}\right]=[3,1]+\left[2,1^{2}\right]
$$

has no geometrical significance.

We may study the geometry of the representation $\left[2,1^{2}\right]$ in a similar fashion, noting from Table 2 that only the vertices of the fundamental region are well defined; since $H_{3} \subset H_{5}$, the groups of stability are $H_{2}, H_{4}$ and $H_{5}$ and their conjugates. It may be verified that

$$
g_{2} \times g_{4}=4 g_{1}, g_{2} \times g_{5}=3 g_{1}, g_{4} \times g_{5}=2 g_{1}
$$

and from Table 3

$$
g_{2} \times^{2}=5 g_{1}+2 g_{2}, g_{4} \times^{2}=2 g_{1}+g_{4}, g_{5} \times^{2}=g_{1}+2 g_{5} .
$$

Moreover, these inner products and the $g_{i} \otimes[2]$ and $g_{i} \otimes[3](i=2,4,5)$ interpreted relative to $\left[2,1^{2}\right]$, describe the familiar arrangement of the vertices, mid-edge and mid-face points, of the octahedron, since the rotation group of the octahedron is isomorphic to the representation $\left[2,1^{2}\right]$ of $S_{4}$.

4. Thus it appears that the geometry of the fundamental region of a real irreducible $\lambda$ can be completely described in terms of $g_{i} \times g_{j}$ and $g_{i} \otimes[n]$. In order to clarify further these ideas, consider the relation 


$$
g_{7} \otimes[2]=g_{7}+g_{9}+g_{2}
$$

which is more interesting than $g_{3} \otimes[2]=g_{3}+g_{i}$, since the octahedron is centrally symmetrical. Denoting the mid-point of the edge $i j$ of the tetrahedron by $P_{i j}$, we have three possibilities: i) pairing $P_{12}$ with $P_{12}$ yields $g_{7}$; ii) pairing $P_{12}$ with $P_{34}$ allows an extra symmetry, since $H_{i}$ is invariant under (1324), which yields $g_{9}$; iii) pairing $P_{12}$ with $P_{13}$ yields a point on the edge of the fundamental region and so $g_{2}$. Since no point is invariant under $H_{i}$ and also (1324), $g_{9}$ does not register in either $[3,1]$ or $\left[2,1^{2}\right]$.

In particular, if $H_{i}$ is a group of stability with $m_{i}^{\lambda}=1$, considerations of linear dependence imply that

4.1 $g_{i} \otimes[n]$ yields every $g_{j}$ with $m_{j}^{\lambda}=1$, for $n$ sufficiently large.

The geometry of the octahedron suggests immediately that $g_{5} \otimes[3]$ yields $g_{4}$ but we must go to $g_{2} \otimes[4]$ and $g_{4} \otimes[4]$ to obtain $g_{5}$, as may readily be verified.

These ideas may be extended to apply to complex $\lambda$ but we shall not consider such a genralization here.

\section{REFERENCES}

[1] R. Brauer, On finite groups and their characters. Bull. Amer. Math. Soc. 69 (1936), $125-130$.

[2] R. Brauer, A note on theorems of Burnside and Blichfeldt. Proc. Amer. Math. Soc. 15 (1964), 31-34.

[3] W. Burnside, Theory of groups of finite order, 2nd. ed. (Cambridge, 1911).

[4] G. de B. Robinson, On the fundamental region of an orthogonal representation of a finite group. Proc. London Math. Soc. 43 (1937), 289-301.

[5] G. de B. Robinson, Representation theory of $S_{u}$, (Toronto, 1961).

[6] R. Steinberg, Complete sets of representations of algebras. Proc. Amer. Math. Soc. 13 (1962), 746-747.

[7] H. Weyl, Classical Groups, (Princeton, 1946).

University of Toronto 
